if the boundary $b$ is displaced by an amount $\delta n=\epsilon \rho(s)$ in the direction of the inner normal. Later the more general variational method of M. Schiffer and the numerous results obtained by means of it are discussed.

While in the preceding chapters the existence of the Green's function, the Neumann function, etc., is assumed (which is permissible since the proofs can be found in many places in the literature), this borrowing is now dispensed with and the slit mapping is carried through without this assumption.

In Chapter 10 it is shown that the methods applied in the preceding chapters can also be applied to the solutions of partial differential equations of elliptic type. Here there are unexpected results from recent investigations of Bergman and of Bergman and Schiffer. The chapter ends with a treatment of the equation of elasticity, $\Delta \Delta \phi=0$, in order to show how the process must be modified for equations of higher order.

The final chapter is concerned with functions of two complex variables and the analytic (pseudo-conformal) mappings generated by them. It is written for readers who are already familiar with the foundations of the theory of functions of several complex variables. First special regions (bicylinder, hypersphere, etc.) are treated; then the orthogonal functions are introduced for arbitrary schlicht bounded domains, and the mappings on representative regions by means of minimal functions and the invariant metric are set up. Finally the author discusses regions with distinguished boundary surfaces, the corresponding Bergman integral representations, and the "extended classes of functions." The choice of the topics in this chapter is perhaps somewhat too much oriented in the direction of the author's own extensive publications.

However, the book as a whole gives a distinguished introduction to the theory of orthogonal functions with its abundance of new results.

\title{
H. BEHNKE
}

Theorie des distributions. By Laurent Schwartz. (Publications de l'Institut de Mathématique de l'Université de Strasbourg, nos. 9 and 10; Actualités Scientifiques et Industrielles, nos. 1091 and 1122.) Vol. I, 1950, 148 pp. Vol. II, 1951, 169 pp.

In Euclidean $E_{k}$ we consider a general function $\varphi(x)=\varphi\left(x_{1}, \cdots, x_{k}\right)$ which is defined and infinitely differentiable everywhere and is zero outside a bounded domain $D=D_{\varphi}$, and, as in a previous context, we call such a function a testing function. Next, if $F=F(x)$ is a fixed 
function in $E_{k}$ which at first is continuously differentiable of order not less than $n$, then, by partial differentiation, we obtain, for $k=1$, for the integral

$$
\int_{-\infty}^{\infty} \varphi \frac{d^{n} F}{d x^{n}} d x
$$

the value

$$
(-1)^{n} \int_{-\infty}^{\infty} F \frac{d^{n} \varphi}{d x^{n}} d x
$$

and for general $k$ we obtain for

$$
\int_{E_{k}} \varphi \frac{\partial^{n_{1}+\cdots+n_{k} F}}{\partial^{n_{1}} x_{1} \cdots \partial^{n_{k}} x_{k}} d v_{x}
$$

the value

$$
(-1)^{n_{1}+\cdots+n_{k}} \int_{E_{k}} F \frac{\partial^{n_{1}+\cdots+n_{k}} \varphi}{\partial^{n_{1}} x_{1} \cdots \partial^{n_{k}} x_{k}} d v_{x}
$$

Now, for the computation of the integrals (2), (4) the function $F$ need not be differentiable and this leads to defining the symbols (1), (3) for testing functions $\varphi$ (in terms of their values (2), (4)) even if the differentiation on $F$ cannot be carried out literally. Such generalized integrals have been long in developing, and their systematic use was the very basis for the theory of generalized Fourier transforms as presented in the reviewer's book Fouriersche Integrale, 1932.

Now, the author proceeds as follows. On the distributive space of testing functions $\{\varphi\}$ introduce a limit topology by calling a sequence $\left\{\varphi_{j}\right\}$ convergent to $\varphi$ if the given functions all vanish outside a joint bounded domain $D_{0}$, and if we have, uniformly in $E_{k}$,

$$
\lim _{j \rightarrow \infty} \frac{\partial^{n_{1}+\cdots+n_{k}} \varphi_{j}}{\partial^{n_{1}} x_{1} \cdots \partial^{n_{k} x_{k}}}=\frac{\partial^{n_{1}+\cdots+n_{k}} \varphi}{\partial^{n_{1}} x_{1} \cdots \partial^{n_{k} x_{k}}}
$$

for all $n_{1} \geqq 0, \cdots, n_{k} \geqq 0$; and, more generally, call the sequence $n$-convergent if (5) holds for $n_{1}+\cdots+n_{k} \leqq n$. Obviously, (4) represents for every continuous $F$ a distributive functional on $\{\varphi\}$ which is continuous in this topology, and for given $n$ even $n$-continuous; and the author establishes the following converse which is the actual content of volume I. Given any continuous functional of this kind, then corresponding to any bounded $D_{0}$ there exist an $F$ and a system $\left\{n_{j}\right\}$ such that for those testing functions which vanish outside $D_{0}$, the 
functional can be represented by the integral (4), and is thus also $n$-continuous in $D_{0}$, for some finite $n$.

In the first half of volume II this theorem is adapted to a Euclidean multi-torus, a testing function being any function

$$
\varphi(x)=\sum a_{r_{1}} \cdots r_{k} e^{i\left(r_{1} x_{1}+\cdots+r_{k} x_{k}\right)}
$$

which is infinitely differentiable, without its being required to vanish anywhere; it can also be characterized by the necessary and sufficient condition that for every $n>0$ we have

$$
a_{r_{1}} \cdots r_{k}=O(|r|-n)
$$

as $|r| \rightarrow \infty$, where $r=\left(r_{1}^{2}+\cdots+r_{k}^{2}\right)^{1 / 2}$. Now, the topology for testing functions being introduced as before, the result is that any continuous functional is automatically $n$-continuous for some $n=n_{0}$, and that the functional has the value

$$
\sum_{(r)} a_{r_{1}} \cdots r_{k} t_{r_{1}} \cdots r_{k}
$$

where the "multiplier" $t_{r_{1}} \ldots r_{k}$ is the value of the functional for the particular testing function $e^{i\left(r_{1} x_{1}+\cdots+r_{k} x_{k}\right)}$. We may then also associate with the functional itself the unique expansion

$$
\sum t_{r_{1}} \cdots_{r_{k}} e^{i\left(r_{1} x_{1}+\cdots+r_{k} x_{k}\right)}
$$

and a sequence of multipliers will constitute such an expansion if and only if for some $n_{0}>0$ we have

$$
t_{r_{1} \cdots r_{k}}=O\left(|r|^{n_{0}}\right)
$$

as $|r| \rightarrow \infty$. And finally it follows that the series (9) is convergent by arbitrary partial sums of itself in the (dual) topology of the space of functionals. The author rather prides himself on this last statement, but within the given context if amounts only to stating that if (10) holds, then for some other exponent $n=m$ we have

$$
\sum^{\prime} \frac{\left|t_{r_{1} \cdots r_{k}}\right|}{|r|^{m}}<\infty,
$$

as we indeed do have for $m=n_{0}+2$, say. We might perhaps recall that in the $L_{2}$-theory, the multiple Fourier series likewise converges by arbitrary partial sums in the dual topology there given.

The second half of volume II is given over to generalized Fourier integrals, and there the analysis had always been very much subtler, and has so remained.

If $f(x)$ in $(-\infty, \infty)$ is $O\left(|x|^{-2}\right)$ at infinity, say, then it has a 
transform

$$
g(\alpha)=\frac{1}{2 \pi} \int_{-\infty}^{\infty} f(x) e^{-i x \alpha} d x
$$

from which it can be re-obtained as

$$
f(x) \sim \int_{-\infty}^{\infty} e^{i x \alpha} g(\alpha) d \alpha .
$$

If, now, for any $n>0, g_{n}(\alpha)$ denotes an $n$th indefinite integral of $g(\alpha)$, then we write for (12)

$$
f(x) \sim \int_{-\infty}^{\infty} e^{i x \alpha} \frac{d^{n} g_{n}(\alpha)}{d \alpha^{n}} d \alpha,
$$

and in a suitable normalization we have

$$
\begin{aligned}
2 \pi g_{n}(\alpha)= & \int_{-1}^{1} f(x) \frac{e^{-i x \alpha}-\sum_{k=0}^{n-1} \frac{(-i x \alpha)^{k}}{k !}}{(i x)^{n}} d x \\
& +\left(\int_{-\infty}^{-1}+\int_{1}^{\infty}\right) f(x) \frac{e^{-i x \alpha}}{(-i x)^{n}} d x .
\end{aligned}
$$

Now, this expression may be calculated even if $f(x)$ does not vanish at infinity; it suffices that we have $f(x)=O\left(|x|^{l}\right)$ for some finite $l$, and (14) may be then introduced, for $n \geqq l+2$ at any rate. The resulting function $g_{n}(\alpha)$ will not be differentiable any more, but we neverthe less envisaged the relation (13) in a symbolic fashion, and these were the generalized Fourier integrals in our book cited. Actually we gave a first but different systematic version in an earlier paper, ${ }^{1}$ and we also gave an application to almost periodic functions which ought to be generalized to several variables. ${ }^{2}$ In these earlier papers we followed a lead of Norbert Wiener in considering functions which were locally $L_{2}$-integrable instead of $L_{1}$-integrable, and this made the theory so much more complicated than the one we arrived at eventually in Fouriersche Integrale that we even did not emphasize them

1 Darstellung reellvariabler und analytischer Funktionen durch Verallgemeinerte Fourier- und Laplace-Integrale, Math. Ann. vol. 97 (1927) pp. 635-662.

2 Über gewisse Differential- und allgemeinere Gleichungen, deren Lösungen fastperiodisch sind I, II, III, Math. Ann. vol. 102 (1929) pp. 489-504; vol. 103 (1930) pp. 588-597; vol. 104 (1931) pp. 579-587.-For a later application of generalized Fourier integrals see also our paper jointly with R. P. Boas, Jr.: Closure theorems for translations, Ann. of Math. (2) vol. 39 (1938) pp. 287-300. 
afterwards; but for the present-day re-study of the entire topic they may contain view points yet explorable.

Now, turning again to our relation (13) we note that the author goes a step further than we did and he also differentiates the function $f(x)$ itself symbolically, thus

$$
\frac{d^{p} f(x)}{d x^{p}} \sim \int_{-\infty}^{\infty} e^{i x \alpha}(i \alpha)^{p} \frac{d^{n} g_{n}(\alpha)}{d \alpha^{n}} d \alpha .
$$

At first sight this still leaves the two sides unsymmetric in that, seemingly, on the right side the integrand is

$$
(i \alpha)^{p} \frac{d^{n} g_{n}(\alpha)}{d \alpha^{n}}
$$

with the unbalancing factor $(i \alpha)^{p}$ in front. It turns out however that for a suitable $G(\alpha)$ and $m$ this can be symbolically written as

$$
\frac{d^{m} G(\alpha)}{d \alpha^{m}}
$$

and in this way the author arrives at a symmetric Fourier transformational reciprocity between symbols

$$
\frac{d^{p} F(x)}{d x^{p}}, \quad \frac{d^{m} G(\alpha)}{d \alpha^{m}}
$$

as it were, the functions $F(x), G(\alpha)$ being arbitrary continuous functions in $(-\infty, \infty)$ which are $O\left(|x|^{a}\right)$ and $O\left(|\alpha|^{n}\right)$ at infinity, the indices $p, m, q, n$ being unrestricted.

The operational foundation for this reciprocation is as follows. If $\varphi(x)$ is a testing function as before, then its transform

$$
\psi(\alpha)=\frac{1}{2 \pi} \int_{-\infty}^{\infty} \varphi(x) e^{-i x \alpha} d x
$$

will not vanish outside a finite interval. But if we assume only that $\varphi(x)$ is infinitely differentiable and that itself and each derivative is $O(|x|-l)$ at infinity for every finite $l$, then its transform $\psi(\alpha)$ has the same property. Thus, the class of these functions is self-inversible under Fourier transformation, and we shall call them semitesting functions. Now (11) and (17) imply by "convolution"

$$
\frac{1}{2 \pi} \int_{-\infty}^{\infty} f(x) \varphi(x) d x=\int_{-\infty}^{\infty} g(\alpha) \psi(-\alpha) d \alpha
$$


and this suggests that for a pair of reciprocal symbols (16) we ought to have the relation

$$
\frac{1}{2 \pi} \int_{-\infty}^{\infty} \frac{d^{p} F(x)}{d x^{p}} \varphi(x) d x=\int_{-\infty}^{\infty} \frac{d^{n} G(\alpha)}{d \alpha^{n}} \psi(-\alpha) d \alpha .
$$

This is indeed so, provided we interpret the symbol

$$
\int_{-\infty}^{\infty} \frac{d^{p} F(x)}{d x^{p}} \varphi(x) d x
$$

and also the right side in (19), in the following manner. In the space of semi-testing functions we introduce a topology which is based on the following limit concept: a sequence $\left\{\varphi_{j}\right\}$ converges to 0 if for every $p \geqq 0, q \geqq 0$ the sequence

$$
x^{p} \frac{d^{q} \varphi_{j}(x)}{d x^{q}}
$$

is convergent to 0 , as $j \rightarrow \infty$, uniformly in $(-\infty, \infty)$. This topology is different from the previous one even if applied to testing functions proper, and we now consider among the functionals originally given only such ones as are continuous with respect to the new topology of the testing functions as well. Such a functional can be extended to the semi-testing functions and remains continuous over them, and it is such a functional which can be represented in the form (20). These are the "distributions temperrées" of the author and they can be associated in pairs in such a manner that (19) shall hold for any pair of semi-testing functions themselves reciprocal, and two distributions thus associated are Fourier transforms of one another.

The author does all this for several variables as well, the class of semi-testing functions being again self-inversive. We note however that the resulting self-inversiveness of the class of distributions, interesting as it is, is only the "dual" to the self-inversiveness of the semi-testing functions themselves, which latter self-inversiveness is a rather obvious phenomenon and, for instance, cannot compare in subtlety to the self-inversiveness of the Plancherel transforms, say, where a natural norm is preserved as well.

The author also gives an extension of the reviewer's theorem on positive definite functions

$$
P\left(x_{1}, \cdots, x_{k}\right)=\int_{E_{k}} e^{i\left(x_{1} \alpha_{1}+\cdots+x_{k} \alpha_{k}\right)} d T\left(\alpha_{1}, \cdots, \alpha_{k}\right)
$$

for positive masses $d T \geqq 0$, from such ones for which the total mass is 
bounded

$$
\int_{E_{k}} d T(\alpha)<\infty
$$

to more general ones for which the total mass is only "slowly" bounded, that is

$$
\int_{E_{k}} \frac{d T(\alpha)}{\left(1+\alpha_{1}^{2}+\cdots+\alpha_{k}^{2}\right)^{l}}<\infty
$$

for some $l$. The transform $P(x)$ is then not a function but a distribution of the author's kind, and he adapts our necessary and sufficient condition of positive-definiteness to his distribution so as to secure again the representation (21). But we might state that, contrary to what the author seems to think, we ourselves gave the theorem not only for one variable in Fouriersche integrale, but also for several variables immediately afterwards. ${ }^{3}$

A dominant analytical tool in the work is a certain "smoothing" process (in French "régularisation") which is used both to localize pieces of a spectrum or of a functional, and to approximate to a distribution by a function. As an analytical tool it is older than sometimes realized and it has been constantly used by us both for generalized Fourier integrals and almost periodic functions; and the closely related "partition of unity," so-called, which is gaining in importance in the cohomology theory of differential forms was introduced for the first time for just such a purpose in our note: Remark on the theorem of Green, Duke Math. J. vol. 3 (1937) pp. 333-338. And as regards the novelty of introducing "distributions" which are more general than Stieltjes integrals, say, we think that the credit for it ought to be assigned to Riemann who in his paper on trigonometric series interprets a series

$$
\sum_{1}^{\infty}\left(\lambda_{n} \cos n x+\mu_{n} \sin n x\right)
$$

with only $\lambda_{n} \rightarrow 0, \mu_{n} \rightarrow 0$, as a symbol

$$
\frac{d^{2} F}{d x^{2}}
$$

where $F(x)$ is defined as the uniformly convergent series

${ }^{3}$ Monotone Funktionen, Stieltjessche Integrale und harmonische Analyse, Math. Ann. vol. 108 (1933) pp. 378-410. 


$$
-\sum_{1}^{\infty} \frac{\lambda_{n} \cos n x+\mu_{n} \sin n x}{n^{2}}
$$

and then "convolves" the series (22) with that of a testing function in the appropriate manner.

We have recounted all this with a view to suggesting that it would not be easy to decide what the general innovations in the present work are, analytical and even conceptual, and that it is in order to appraise the value of the book by its specific results, such as we have extracted above; and of such let the author produce many more, by all means.

\section{S. BOCHNER}

Tables relating to Mathieu functions. Characteristic values, coefficients, and joining factors. Prepared by the Computation Laboratory of the National Applied Mathematics Laboratories, National Bureau of Standards. New York, Columbia University Press, 1951.48+278 pp. $\$ 8.00$.

Since its foundation (January 1938) the New York Unit of the Computation Laboratory of the National Applied Mathematics Laboratories, a division of the National Bureau of Standards-until July 1947 it was called the Mathematical Tables Project-has been very active in producing extensive and accurate numerical tables of important mathematical functions. Besides a series of tables of the elementary transcendents, they have published almost a dozen tables relating to the higher transcendents such as sine, cosine, and exponential integrals, probability functions, Bessel functions, Legendre functions. No matter how high-speed electronic calculating machinery may be further developed, applied mathematicians will always owe a great debt to these and other table-makers.

This particularly holds for the fascinating tables under review. As Professor Erdélyi emphasizes in the foreword, the comparatively slender numerical material available for Mathieu functions shows the urgency of the task undertaken by the National Bureau of Standards. The more so, since several important problems of applied mathematics and theoretical physics involving Mathieu functions have so far received only little attention because of lack of adequate numerical data. These problems include all types of vibrational, wave and diffusion problems connected with ellipses or elliptic cylinders, as well as stability investigations of various mechanical systems, the theory of frequency modulation, and loud-speaker theory. 